\title{
What is a knowledge ecosystem?
}

While reading an article for an education class I am taking, I found myself exploring the idea of knowledge. The article related the concept to an ecosystem. As a biologist learning about teaching, I wondered how this author would do the reverse, utilizing biology from an educator's perspective.

Franklin Becker's article, "Organisational ecology and knowledge networks" (Dev. Learning Organizations 21; 2007; http:// www.emeraldinsight.com/journals.htm ?articleid $=1621719$ \&show $=$ html), first discusses the need for employees to be "knowledge workers" in what is now the "information age." Although it's a few years old, the concepts nevertheless resonate within the lab animal science field. Animal physiological data, protocol specifications, regulatory requirements, equipment SOPs, etc. are all good examples of information we need to know. In my training chapter (in Management of Laboratory Animal Care and Use Programs; CRC Press, Boca Raton, FL, 2001), I categorized knowledge as funto-know, good-to-know and need-to-know. The latter category includes knowledge that relates very specifically to our jobs and tends to be the priority in training sessions.

An important objective of Becker's article was to point out how difficult it is for organizations to manage their copious amounts of knowledge, some of it inherent to the business and the rest gained through research. He specifically addressed the corporate world, but his comments certainly apply to vivaria. He describes how the concepts of ecology and biology can be applied to the collection of business facts and the dissemination of information and knowledge. This application of ecology is much like the paradigm that we expect our institutional trainers to create and cultivate. In the same way that scientists attempt to understand the obvious and subtle networks of communication in ecosystems, trainers must try to recognize the pathways of learning and knowledge exchange. For example, physiological data transfers along biological channels (e.g., nerves); similarly, learning may transfer along technological channels (e.g., audiovisual media). Information is also directly passed via face-to-face exchanges, such as behavioral displays among animal conspecifics in ecosystems or during conferences for training humans. By either means, there is usually an effective sharing of what needs to be known.

Becker cites another author who defines knowledge as "the meaningful links people make in their minds between information and its application in action in a specific setting." I would argue that job assignments, promotions, research publications and regulatory decisions are all manifestations of the application of knowledge in biomedical research.

Trainers train colleagues-animal care providers, investigators, IACUC members, etc.-providing information, facts and processes, so that they can do their jobs. We assess the training by testing or evaluating the trainees through certification exams or by having more experienced persons observe their proficiency at tasks. We accept that the ability of the trainee to perform well on the tests is attributable to his or her retention of the knowledge. [The ideas of assessment and evaluation have long been discussed in other disciplines but only recently have come to laboratory animal science. That will be a topic for future columns.]

Trainers are aware that knowledge is not swallowed like a pill to be digested and absorbed (if only it were that easy!). Instead, packaging the facts with information that frames them, or giving the trainee a lens through which to view and understand them, can enhance the learning and, as importantly, the retention of said facts. Thus, watching a necropsy (in the interest of the $3 \mathrm{Rs}$, perhaps pre-recorded) that teaches about body structure, organs and systems may be more effective as a knowledge or learning process than staring at anatomical charts. In other 'biological' words, the ecosystem adapts.
Becker includes five propositions in his organizational ecology for learning. These conform to the idea of "relevant purpose," which is that which the trainee wants to know or is eager to learn. It relates to the need and motivational principles of psychologist Abraham Maslow and adult educator Malcolm Knowles. Becker calls them design factors in learning but incorporates ecological terms. Here, I've listed them and briefly interpreted them for vivarium learning:

Eco-diversity. Provide various settings and physical niches for learning. Like animals that move around, learners should go beyond the break room for training purposes.

Spatial transparency. In the wild, organisms have a sense of where they are in the ecosystem. Enable all staff members (cage-wash, veterinary, investigators, etc.), as trainees, to see and hear one another.

Functional inconvenience. Chicks are pushed out of the nest in order to learn to survive. Push staff to encounter one another to learn and work together (e.g., participate in lunch-and-learn sessions, attend conferences together, care for animals simultaneously in the holding rooms).

Human scale. Ecosystems have niches. Create smaller or more intimate spaces and departments to encourage learning (crosstrain and train one-on-one as well as in conference rooms).

Neutral zones. Animals observe and learn from one another, both within and outside of their species. Minimize the hierarchical attitudes of one group not able to learn from others (e.g., consider whether the enrichment tech can talk to the neurobehaviorist).

Becker as an educator acknowledges that not all organizations will have the same learning environments and ecology. Similarly, lab animal trainers working in biology can look at their own ecosystems and make modifications to ensure successful transfer of knowledge. Voilà... knowledge ecosystem defined! 